\title{
Analysis of a field conjugation adaptive array for coherent free-space optical links
}

\author{
Aniceto Belmonte ${ }^{1}$ and Joseph M. Kahn ${ }^{2}$ \\ 1. Technical University of Catalonia, Department of Signal Theory and Communications, 08034 Barcelona, Spain. \\ 2. Stanford University, Department of Electrical Engineering, Stanford, CA 94305, USA. \\ belmonte@tsc.upc.edu
}

\begin{abstract}
We study the performance of diversity combining techniques applied to synchronous laser communication through the turbulent atmosphere. We assume that a single informationbearing signal is transmitted over statistically independent fading channels, and that the multiple replicas are combined at the receiver to improve detection efficiency.

(C) 2009 Optical Society of America

OCIS codes: (010.1330) Atmospheric turbulence; (060.4510) Optical communications, Coherent receivers; Diversity combining.
\end{abstract}

Evaluating the performance of a heterodyne receiver in the presence of atmospheric turbulence is generally difficult because of the complex ways turbulence affects the coherence of the received signal that is to be mixed with the local oscillator. Light propagated through a turbulent atmosphere contains speckle which will be present at the detector surface. Therefore, illuminating a single-element detector with a uniform LO beam will produce mismatch of the amplitudes and phases of the two fields resulting in a loss in downconverted power. The downconverted heterodyne power is maximized when the spatial field of the received signal matches that of the local oscillator.

As an alternative to a single monolithic-aperture coherent receiver with a full-size collecting area, a large effective aperture can be achieved by combining the output signal from an array of smaller receivers (see Fig. 1). The advantage of a coherent array in terms of the coupling efficiency is that the number of turbulence speckles over each subaperture in the array is much smaller than it would be over a single large aperture. Because each receiver can now be smaller than the scale on which the signal wavefront varies, the local oscillator phase can be matched to the signal to achieve effective coherent reception. Output signals from these receivers can then be combined electronically to improve the detection statistics. In general, the performance of a combined system should improve with an increasing number of receivers and, consequently, given a fixed collecting area, the combined system can offer superior performance. It is important to note that diversity combining consists of receiving redundantly the same information signal over two or more fading channels and to exploit the low probability of concurrence of deep fades in all the diversity channels. We define a general model for the output SNR of diversity systems over correlated fading channels. For systems where the atmospheric fading on the branches is correlated or dependent, we can solve the problem by transforming it into an independent problem using the technique of spatial whitening.

We consider two types of receive diversity combining. First we assume the receiver has knowledge of the instantaneous channel state, making perfect maximal ratio combining (MRC) diversity possible. In this diversity scheme, the receiver co-phases the intermediate signals, adjusts their amplitudes separately, and sums them to obtain a composite signal with improved SNR. The rate at which phase and amplitude must be adjusted will be dictated by the rate at which the atmospheric turbulence fluctuates, generally no higher than $1 \mathrm{kHz}$. The MRC receiver is the optimal combining technique in that it yields a carrier with the highest mean SNR and lowest SNR fading. The optimum electronic gain for each receiver should be proportional to the received signal field amplitude. Note that when the electronic gains and phase delays are back-propagated into the LO, the optimum gain and phase adjustments would result in an amplitude and phase match of this synthetic LO field to the distorted signal field. Then we consider the case when selective combining (SC) diversity schemes is used. A selection receiver is quite different from the coherent MRC summing receiver as the single strongest intermediate signal is switched to the output, while all the other array element signals are discarded. Although this simple diversity technique clearly does little to improve the mean SNR value of the collected signal, it does provide a reduction in the signal fading.

In this work, we analyze the spectral efficiency of optical communication over the clear turbulent atmosphere. Defined as the average transmitted data rate per unit bandwidth for a specific average transmit power and probability of outage or error, achievable spectral efficiency is an important performance measure for free-space optical communication systems. As the use of multilevel phase modulation schemes increase spectral efficiency by sending multiple bits per symbol, optical coherent reception provides an alternative to direct detection schemes for freespace optical communication applications.

Figures 2 and 3 show the results of this analysis. Figure 2 considers the effect of aperture diameter on the $\varepsilon$ outage spectral efficiency. It presents the spectral efficiency as a function of the normalized aperture $D / r_{0}$ for a 


\section{LSTuA1.pdf}

constant phase coherence length $r_{0}$. In (a), MRC combining is employed. In (b), a SC combiner is considered. In all cases, the outage probability is small and fixed at $\varepsilon=0.001$, and the channel capacity per unit bandwidth is shown for different values of the number $L$ of combiner independent branches. The case $L=1$ corresponds to no receive diversity. The area $\pi D^{2}$ describes the combined, multi-aperture system equivalent aperture. When no receive diversity is considered, $D$ equals the receiver aperture diameter. If a $L$-aperture system is analyzed, each one of the aperture diameters equals $D / \sqrt{ } L$. For the smallest aperture considered, we assume $\gamma_{0}$ equal to 10 photons-per-symbol. For any other aperture diameter, the value of $\gamma_{0}$ is proportional to $D^{2}$. We study the outage channel capacity as a function of several parameters: the average signal-to-noise ratio (photons) per bit, the strength of atmospheric turbulence, the receiver aperture diameter $D$, and the number $\mathrm{L}$ of combiner branches. Turbulence effects are described by two parameters. Fried's coherence length $r_{0}$ describes the coherent diameter of the distorted wavefront phase. The scintillation index $\sigma_{\beta}$ describes the intensity of amplitude fluctuations. In our modeling, we have chosen typical values of these two parameters. While our model can be applied to various modulation and detection methods, here, we have shown the performance of PSK, QAM, PAM, and DPSK using coherent detection in the presence of additive white Gaussian noise (AWGN). Atmospheric fading leads to serious degradation in the receiver sensitivity measured in terms of received photons per symbol, resulting in either a higher error rate or a higher required transmit power for a given multilevel modulation technique. In general, the performance of an array signalcombining system should improve with an increasing number of receivers and, consequently, given a fixed collecting area, the combined system can offer superior performance.

Note the diminishing capacity returns that are obtained as the number of branches increases. Although as we increase $L$ the outage spectral efficiency improves appreciably, the greatest improvement is still obtained in going from single- to two-branch combining. In any case, even for such small outage probability $\varepsilon=0.001$, a multiple receiver system with $L=8$ apertures reach an outage spectral efficiency of over $10 \mathrm{bits} / \mathrm{s} / \mathrm{Hz}$, very close to the optimal spectral rate of a single branch AWGN channel as defined by Shannon. When a single $(L=1)$ or dual $(L=2)$ receiver system is considered, the existence of an optimal aperture diameter in coherent free-space links is apparent. This optimal aperture diameter maximizes the $\varepsilon$-outage capacity. When the aperture is larger than the optimal value, phase distortion cannot be overcome by the increase in collected power, and we observe a decrease in capacity. For $L>2$ receiver systems, or under strong scintillation conditions, no optimal value can be identified. More detailed results and comments on our analysis will be presented at the meeting.

The research of Aniceto Belmonte was funded by the Spanish Department of Science and Technology MCYT Grant No. TEC 2006-12722

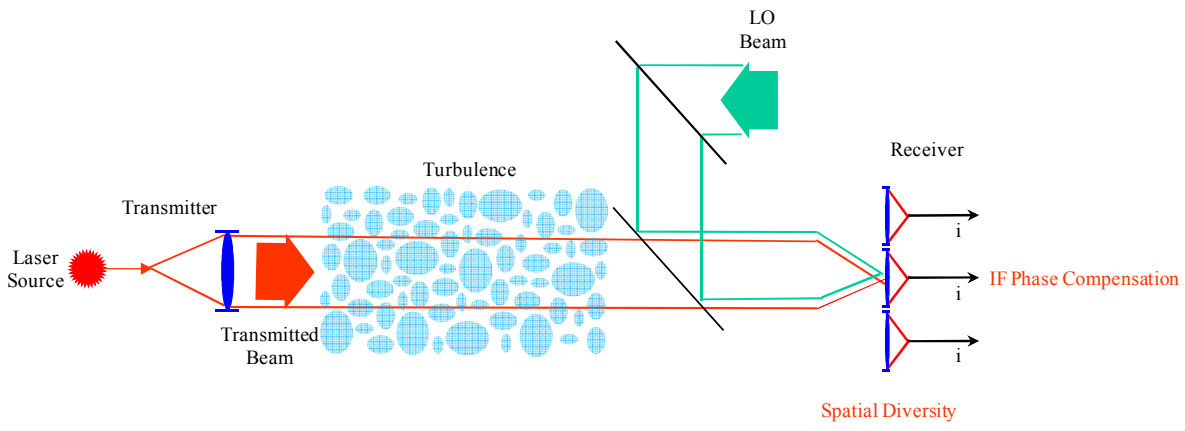

Fig. 1. A coherent free-space optical communication system is affected by the presence of atmospheric turbulence in many ways. First, turbulence induces a decrease of the mean received power level, which translates into a diminished signal-to-noise ratio in the receiver. Second, amplitude scintillation and phase distortion in the receiver plane act as intense sources of noise distorting the quality of the optical signal available for processing. All of them add together to deteriorate the overall communication performance of the optical systems. Coherent array receivers could be considered to alleviate the deteriorating effects of atmospheric turbulence in the performance of coherent receivers in free-space optical communications.. 


\section{LSTuA1.pdf}
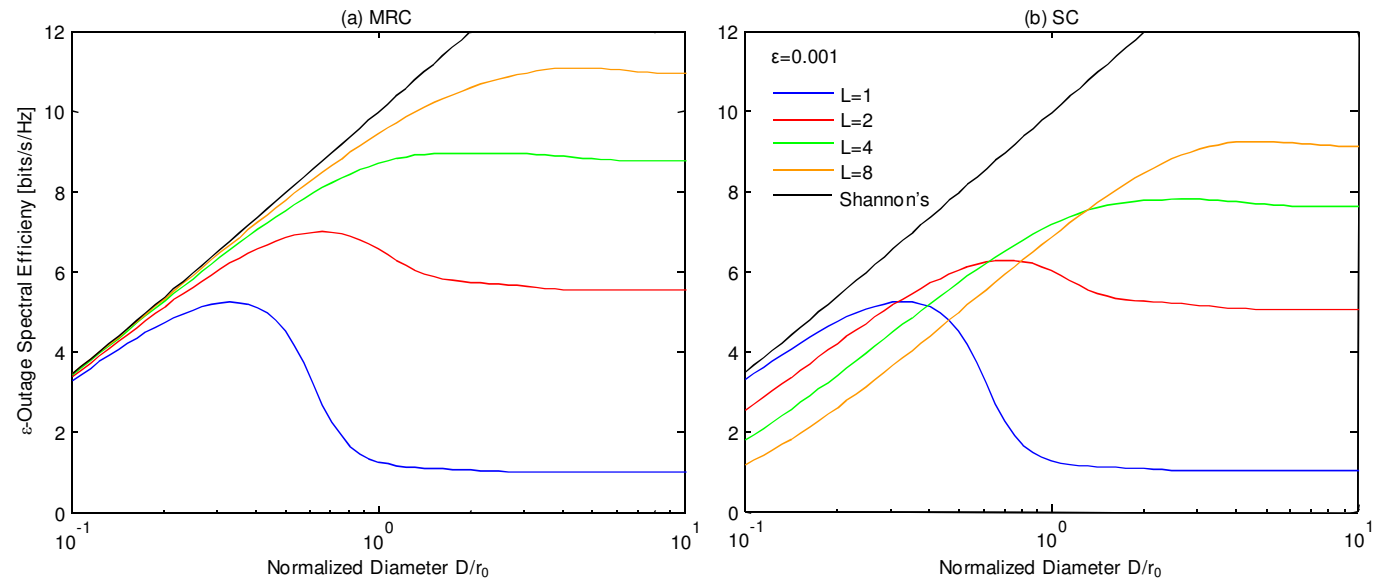

Fig. 2. $\varepsilon$-outage spectral efficiency vs. normalized receiver aperture diameter $D / r_{0}$ for coherent detection and AWGN. In (a), MRC combining is employed. In (b), a SC combiner is considered. In all cases, the outage probability is fixed at $\varepsilon=0.001$, and the channel capacity per unit bandwidth is shown for different values of the number $\mathrm{L}$ of combiner branches. The case $\mathrm{L}=1$ corresponds to no receive diversity (blue lines). The area $\pi \mathrm{D}^{2}$ describes the combined, multi-aperture system equivalent aperture. When no receive diversity is considered, $\mathrm{D}$ equals the receiver aperture diameter. The turbulence-free SNR per symbol $\gamma_{0}$ is proportional to the square of the aperture diameter $D$. For the smallest aperture considered, we assume $\gamma_{0}$ equal to 10 photons per symbol. In this plot, we neglect amplitude fluctuations by assuming $\sigma_{\beta}{ }^{2}=0$. In this case, turbulence is characterized by the phase coherence length $r_{0}$. The AWGN Shannon limit is indicated by black lines.
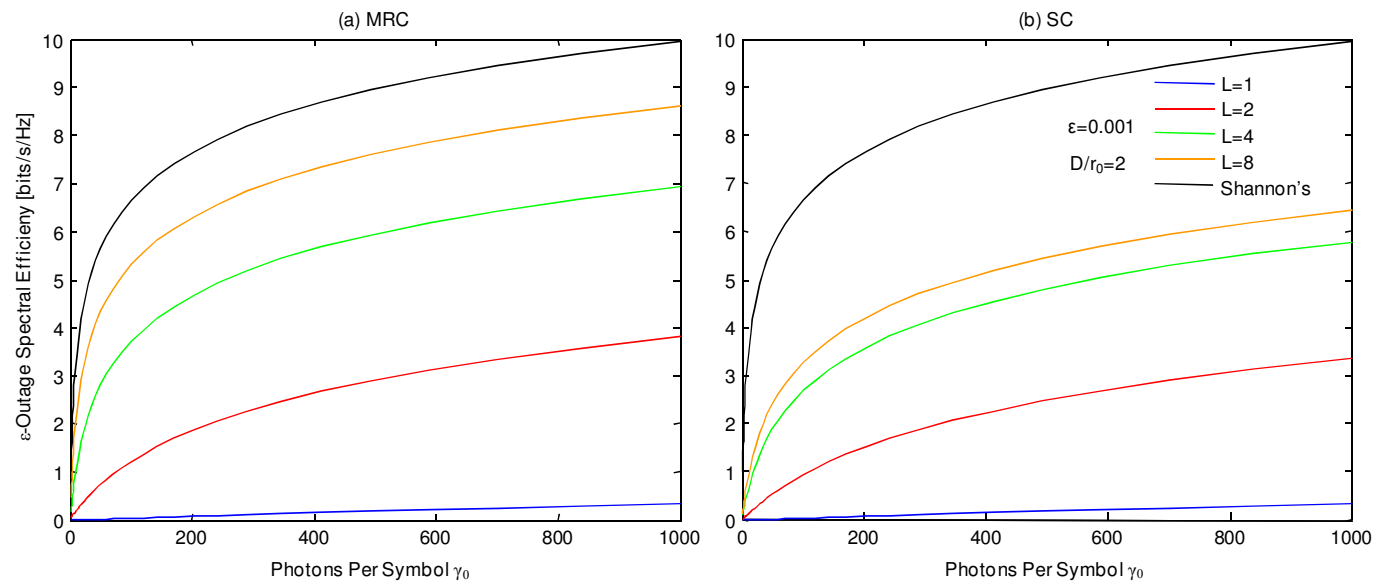

Fig. 3. $\varepsilon$-outage spectral efficiency vs. turbulence-free photons per symbol $\gamma_{0}$ for coherent detection and additive white Gaussian noise (AWGN). In (a), MRC combining is employed. In (b), a SC combiner is considered. In all cases, the outage probability is fixed at $\varepsilon=0.001$, and the channel capacity per unit bandwidth is shown for different values of the number $\mathrm{L}$ of combiner branches. The case $\mathrm{L}=1$ corresponds to no receive diversity (blue lines). Amplitude fluctuations are neglected by assuming $\sigma_{\beta}{ }^{2}=0$. Turbulence is characterized by a moderate phase coherence length $r_{0}$ such as $D / r_{0}=2$. The area $\pi \mathrm{D}^{2}$ describes the combined, multi-aperture system equivalent aperture. The AWGN Shannon limit is indicated by black lines. 